
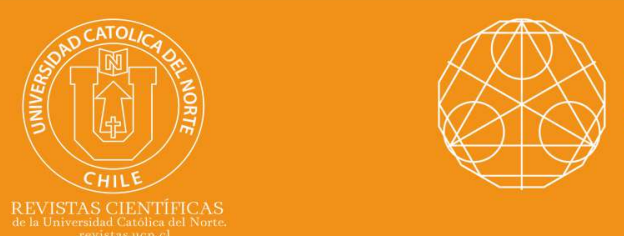

\title{
On reformulated Narumi-Katayama index
}

\author{
Murat Cancan ${ }^{1}$ (1) orcid.org/0000-0002-8606-2274 \\ Nilanjan De ${ }^{2}$ (b) orcid.org/0000-0001-9143-7045 \\ Mehdi Alaeiyan ${ }^{3}$ (1) orcid.org/0000-0003-2185-5967 \\ Mohammad Reza Farahani ${ }^{4}$ (1) orcid.org/0000-0003-2969-4280 \\ ${ }^{1}$ Van Yüzüncü Yıl University, Faculty of Education, Van, Turkey. \\ mcancan@yyu.edu.tr \\ ${ }^{2}$ Calcutta Institute of Engineering \& Management, Dept of Basic Sci and Humanities, WB, Kolkata, India \\ de.nilanjan@rediffmail.com \\ Iran University of Science and Technology, Dept. of Mathematics, Tehran, Iran. \\ 3曰alaeiyan@iust.ac.ir ; 4 mrfarahani88@gmail.com
}

Received: April 2020 | Accepted: August 2020

\section{Abstract:}

A graph is a mathematical model form by set of dots for vertices some of which are connected by lines named as edges. A topological index is a numeric value obtained from a graph mathematically which characterize its topology. The reformulated Narumi-Katayama index of a graph $G$ is defined as the product of edge degrees of all the vertices of $G$ which is introduced in 1984, to used the carbon skeleton of a saturated hydrocarbons. The degree of an edge is given by the sum of degrees of the end vertices of the edge minus 2. In this paper, we compute the reformulated Narumi-Katayama index for different graph operations.

Keywords: Degree; Graph; Graph operations; Reformulated NK-index; Topological indices.

MSC (2020): 05C35, 05C07, 05C40.

\section{Cite this article as (IEEE citation style):}

M. Cancan, N. De, M. Alaeiyan, and M. Reza Farahani, "On reformulated Narumi-Katayama index", Proyecciones (Antofagasta, On line), vol. 39, no. 5, pp. 1333-1346, Oct. 2020, doi: 10.22199/issn.0717-6279-2020-05-0081.

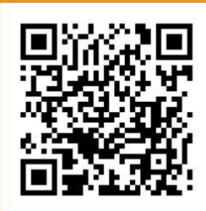

Article copyright: (C) 2020 Murat Cancan, Nilanjan De, Mehdi Alaeiyan, and Mohammad Reza Farahani . This is an open access article distributed under the terms of the Creative Commons Licence, which permits unrestricted use and distribution provided the original author and source are credited. 


\section{Introduction}

Let $G$ be a simple connected graph with vertex set $V(G)$ and edge set $E(G)$. We denote by $d_{G}(v)$ the degree of a vertex $v$ of $G$, which is defined as the number of edges incident to $v$. The first and second Zagreb indices of a graph $G$ are denoted by $M_{1}(G)$ and $M_{2}(G)$ respectively and defined as follows:

$$
M_{1}(G)=\sum_{v \in V(G)} d_{G}(v)^{2}=\sum_{u v \in E(G)}\left[d_{G}(u)+d_{G}(v)\right]
$$

and

$$
M_{2}(G)=\sum_{u v \in E(G)} d_{G}(u) \cdot d_{G}(v) .
$$

These indices were introduced by Gutman et al. in [1] and are among the oldest and most studied degree-based topological indices of graphs.

Narumi and Katayama in 1984 [2], introduced a multiplicative version of degree-based graph invariant for representing the carbon skeleton of a saturated hydrocarbon and named it as a "simple topological index". This invariant is defined as

$$
N K(G)=\prod_{v \in V(G)} d_{G}(v) .
$$

Tomovic and Gutman [3], later renamed this index as "Narumi-Katayama index" or "NK-index". Todeschini et al. in 2010 [4], have introduced the multiplicative version of additive graph invariants and in this regard Eliasi, Iranmaresh and Gutman in 2012 [5], introduced a new multiplicative version of first Zagreb index and called it multiplicative sum Zagreb index, which is defined as

$$
\Pi_{1}^{*}(G)=\prod_{u v \in E(G)}\left[d_{G}(u)+d_{G}(v)\right] .
$$

For further study about multiplicative version Zagreb indices we refer our reader to $[6,7,8,9,10,11]$. We know that, the reformulated Zagreb indices are the edge version of classical Zagreb indices which are introduced by Miličević et al. [12], in 2004 and are defined as

$$
E M_{1}(G)=\sum_{e \in E(G)} d(e)^{2}=\sum_{u v \in E(G)}\left[d_{G}(u)+d_{G}(v)-2\right]^{2},
$$




$$
E M_{2}(G)=\sum_{e \sim f \in E(G)} d(e) d(f)
$$

where $e \sim f$ means that the edges $e$ and $f$ share a common vertex in $G$. For further study, we refer our reader to $[13,14,15,16,17,18,19,20]$. Analogus to reformulated Zagreb indices, in this paper, we introduced a new version of NK-index by replacing vertex degrees with the edge degrees, where degree of an edge $e=u v$ is defined as $d_{G}(e)=d_{G}(u)+d_{G}(v)-2$. Thus, the reformulated NK-index of a graph $G$, denoted by $N K_{E}(G)$ is defined as

$$
N K_{E}(G)=\prod_{e \in E(G)} d_{G}(e) .
$$

In this paper, first we present some preliminary results for the reformulated NK-index and hence derive some explicit for the reformulated NK-index of different graph operations such as join, Cartesian product, Corona product etc. and also consider some special cases.

\section{Preliminary Results.}

As usual, let the complete graph, cycle graph and path graph with $n$ number of vertices are denoted by $K_{n}, C_{n}$ and $P_{n}$ respectively, where as $K_{m, n}$ denotes a complete bipartite graph with $(m+n)$ number of vertices. From the definition of reformulated NK-index, in this section first we derive reformulated NK-index of these standard classes of graph from direct calculation.

\section{Example 1.}

$$
\begin{aligned}
\text { (i) } N K_{E}\left(K_{n}\right) & =(2 n-4)^{\frac{n(n-1)}{2}} \\
(i i) N K_{E}\left(C_{n}\right) & =2^{n},(n \geq 3) \\
(\text { iii }) N K_{E}\left(P_{n}\right) & =2^{n-3},(n \geq 3) \\
\text { (iv) } N K_{E}\left(K_{m, n}\right) & =(m+n-2)^{m n} .
\end{aligned}
$$

Now we recall the well-known A.M.-G.M. inequality, which is required in this paper.

Lemma 1. Let $x_{1}, x_{2}, \ldots \ldots . ., x_{n}$ be non negative numbers, then

$$
\frac{x_{1}+x_{2}+\ldots .+x_{n}}{n} \geq \sqrt[n]{x_{1}, x_{2}, \ldots \ldots ., x_{n}}
$$

with equality if and only if $x_{1}=x_{2}=\ldots=x_{n}$. 
Now using above lemma, in the following we derive upper and lower bounds for reformulated NK-index of a graph $G$.

Proposition 1. Let $G$ ba connected graph with order $n$ and size $m$. Then

$$
N K_{E}(G) \leq\left(\frac{M_{1}(G)}{m}-2\right)^{m}
$$

with equality if and only if $G$ be a regular graph.

Proof. Using lemma 1, we have

$$
\begin{aligned}
\frac{1}{m} \sum_{e \in E(G)} d_{G}(e) & \geq\left[\prod_{e \in E(G)} d_{G}(e)\right]^{\frac{1}{m}} \\
\text { or, } \frac{1}{m}\left(M_{1}(G)-2 m\right) & \geq\left[N K_{E}(G)\right]^{\frac{1}{m}}
\end{aligned}
$$

with equality when $G$ be a regular graph. Hence, the desired result follows.

Proposition 2. Let $G$ ba connected graph with order $n$ and size $m$. Then

$$
N K_{E}(G) \geq \frac{1}{8^{m}} \Pi_{1}^{*}(G)^{2},
$$

with equality if and only if $G$ is a cycle or the star graph on 4 vertices.

Proof. We have from definition of multiplicative sum Zagreb index

$$
\begin{aligned}
\Pi_{1}^{*}(G) & =\prod_{u v \in E(G)}\left[d_{G}(u)+d_{G}(v)\right]^{2} \\
& =\prod_{u v \in E(G)}\left[\left(d_{G}(u)+d_{G}(v)-2\right)+2\right]^{2}
\end{aligned}
$$

So, using lemma 1, we have

$$
\begin{aligned}
\Pi_{1}^{*}(G) & \geq \prod_{u v \in E(G)}\left[2 \sqrt{\left(d_{G}(u)+d_{G}(v)-2\right) \times 2}\right]^{2} \\
& =8^{m} \prod_{u v \in E(G)}\left[d_{G}(u)+d_{G}(v)-2\right] \\
& =8^{m} N K_{E}(G) .
\end{aligned}
$$

From, lemma 1, it is clear that, in the above inequality, equality holds if and only if for every $e=u v \in E(G), d_{G}(e)=2$. Thus, $G$ is either a cycle or the star graph on 4 vertices. Hence, the desired result follows. 


\section{Reformulated NK-index of graph operations:}

In this section, we study some upper bounds of reformulated NK-index under different graph operations. Hence, we find the equality conditions for these inequalities unless stated otherwise, we consider $G_{i}$ connected graph with $n_{i}$ number of vertices and $m_{i}$ number of edges, for $i \in\{1,2\}$.

\section{The join of graphs:}

The join $G_{1}+G_{2}$ of two graphs $G_{1}$ and $G_{2}$ with disjoint vertex sets $V\left(G_{1}\right)$ and $V\left(G_{2}\right)$, and edge sets $E\left(G_{1}\right)$ and $E\left(G_{2}\right)$ is the graph union $G_{1} \cup G_{2}$ together with all the edges between $V\left(G_{1}\right)$ and $V\left(G_{2}\right)$. Thus, $\left|V\left(G_{1}+G_{2}\right)\right|=$ $\left|V\left(G_{1}\right)\right|+\left|V\left(G_{2}\right)\right|$ and $\left|E\left(G_{1}+G_{2}\right)\right|=\left|E\left(G_{1}\right)\right|+\left|E\left(G_{2}\right)\right|+\left|V\left(G_{1}\right)\right|\left|V\left(G_{2}\right)\right|$. The degree of a vertex $v \in V\left(G_{1}+G_{2}\right)$ is given by

$$
d_{G_{1}+G_{2}}(v)=\left\{\begin{array}{l}
d_{G_{1}}(v)+\left|V\left(G_{2}\right)\right|, \text { if } v \in V\left(G_{1}\right) \\
d_{G_{2}}(v)+\left|V\left(G_{1}\right)\right|, \text { if } v \in V\left(G_{2}\right) .
\end{array}\right.
$$

Now, in the following we derive the upper bound of reformulated NKindex of $G_{1}+G_{2}$.

Theorem 1. Let $G_{1}$ and $G_{2}$ be two vertex disjoint connected graphs. Then

$$
\begin{aligned}
N K_{E}\left(G_{1}+G_{2}\right) \leq & {\left[\frac{M_{1}\left(G_{1}\right)}{m_{1}}+2\left(n_{2}-1\right)\right]^{m_{1}}\left[\frac{M_{1}\left(G_{2}\right)}{m_{2}}\right.} \\
& \left.+2\left(n_{1}-1\right)\right]^{m_{2}} \times\left[\frac{2 m_{1}}{n_{1}}+\frac{2 m_{2}}{n_{2}}\right. \\
& \left.+n_{1}+n_{2}-2\right]^{n_{1} n_{2}}
\end{aligned}
$$

In the above inequality, equality holds if and only if both $G_{1}$ and $G_{2}$ are regular graphs.

Proof. The edge set of $G_{1}+G_{2}$ can be partitioned into three subsets, namely $E_{1}=E\left(G_{1}\right), E_{2}=E\left(G_{2}\right)$ and $E_{3}=\left\{e=u v: u \in V\left(G_{1}\right), v \in V\left(G_{2}\right)\right\}$. Then from definition of reformulated NK-index and using lemma 1 , the contribution of the edges of $E_{1}$ is given by 


$$
\begin{aligned}
J_{1} & =\prod_{u v \in E\left(G_{1}\right)}\left\{d_{G_{1}+G_{2}}(u)+d_{G_{1}+G_{2}}(v)-2\right\} \\
& =\prod_{u v \in E\left(G_{1}\right)}\left\{d_{G_{1}}(u)+n_{2}+d_{G_{1}}(v)+n_{2}-2\right\} \\
& \leq\left[\frac{1}{m_{1}} \sum_{u v \in E\left(G_{1}\right)}\left\{\left(d_{G_{1}}(u)+d_{G_{1}}(v)\right)+2\left(n_{2}-1\right)\right\}\right]^{m_{1}} \\
& =\left[\frac{M_{1}\left(G_{1}\right)}{m_{1}}+2\left(n_{2}-1\right)\right]^{m_{1}}
\end{aligned}
$$

In the above inequality, equality holds, if and only if for every $u v \in$ $E\left(G_{1}\right)$ and $x y \in E\left(G_{1}\right)$

$$
d_{G_{1}}(u)+d_{G_{1}}(v)+2\left(n_{2}-1\right)=d_{G_{1}}(x)+d_{G_{1}}(y)+2\left(n_{2}-1\right) .
$$

Similarly, the contribution of the edges of $E_{2}$ is given by

$$
\begin{aligned}
J_{2} & =\prod_{u v \in E\left(G_{2}\right)}\left\{d_{G_{1}+G_{2}}(u)+d_{G_{1}+G_{2}}(v)-2\right\} \\
& =\prod_{u v \in E\left(G_{2}\right)}\left\{\left(d_{G_{2}}(u)+d_{G_{2}}(v)\right)+2\left(n_{1}-1\right)\right\} \\
& \leq\left[\frac{M_{1}\left(G_{2}\right)}{m_{2}}+2\left(n_{1}-1\right)\right]^{m_{2}}
\end{aligned}
$$

In the above inequality, similarly equality holds, if and only if for every $u v \in E\left(G_{2}\right)$ and $x y \in E\left(G_{2}\right)$

$$
d_{G_{2}}(u)+d_{G_{2}}(v)+2\left(n_{1}-1\right)=d_{G_{2}}(x)+d_{G_{2}}(y)+2\left(n_{1}-1\right) .
$$

Again, the contribution of the edges of $E_{3}$ to the reformulated NK-index of join of graphs is given by

$$
\begin{aligned}
J_{3}= & \prod_{\substack{u v \in E\left(G_{1}+G_{2}\right), u \in V\left(G_{1}\right), v \in V\left(G_{2}\right) \\
\\
=}}\left(\prod_{u \in V\left(G_{1}\right)} \prod_{v \in V\left(G_{2}\right)} d_{G_{1}+G_{2}}(u)\right. \\
& d_{G_{1}}(u)+n_{2}+d_{G_{2}}(v)+n_{1}-2
\end{aligned}
$$


Now using lemma 1, we get

$$
\begin{aligned}
J_{3} \leq & {\left[\frac { 1 } { n _ { 1 } n _ { 2 } } \sum _ { u \in V ( G _ { 1 } ) } \sum _ { v \in V ( G _ { 2 } ) } \left(d_{G_{1}}(u)+d_{G_{2}}(v)+n_{1}+n_{2}\right.\right.} \\
& -2)]^{n_{1} n_{2}} \\
= & {\left[\frac{1}{n_{1} n_{2}}\left(2 m_{1} n_{2}+2 m_{2} n_{1}+n_{1} n_{2}\left(n_{1}+n_{2}-2\right)\right)\right]^{n_{1} n_{2}} }
\end{aligned}
$$

It is clear that, in the above inequality equality holds if and only if both $G_{1}$ and $G_{2}$ are regular graphs. Hence, combining the contributions $J_{1}, J_{2}$ and $J_{3}$ we get the desired result as theorem 1 .

Corollary 1. Let $G_{1}$ be a $r_{1}$-regular graph and $G_{2}$ be a $r_{2}$-regular graph, then

$$
\begin{aligned}
N K_{E}\left(G_{1}+G_{2}\right)= & 2^{m_{1}+m_{2}}\left(r_{1}+n_{2}-1\right)^{m_{1}}\left(r_{2}+n_{1}-\right. \\
& 1)^{m_{2}} \times\left(n_{1}+n_{2}+r_{1}+r_{2}-2\right)^{n_{1} n_{2}} .
\end{aligned}
$$

\section{Example 2.}

(i) $N K_{E}\left(C_{n}+C_{m}\right)=2^{m+n}(n+1)^{m}(m+1)^{n}(n+m+2)^{n m}$

Let $G=G_{1}+G_{2}+\ldots . .+G_{k}$ and $n=n_{1}+n_{2}+\ldots . .+n_{k}$ for some positive integer $k(\geq 2)$. Then, the degree of the vertices of $G$ are given by

$$
d_{G}(v)=d_{G_{i}}(v)+\bar{n}_{i} \text {, if } v \in V\left(G_{i}\right)
$$

where, $\bar{n}_{i}=n-n_{i}$ for $1 \leq i \leq k$. Then, using theorem 1 , we get the following result using mathematical induction.

Theorem 2. Let $G_{1}, G_{2}, \ldots . ., G_{k}$ be $k$-number of vertex disjoint graphs. Then

$$
\begin{aligned}
N K_{E}(G)= & \prod_{i=1}^{k}\left[\frac{M_{1}\left(G_{i}\right)}{m_{i}}+2\left(\overline{n_{i}}-1\right)\right]^{m_{i}} \prod_{1 \leq i \leq j \leq k}\left[\frac{2 m_{i}}{n_{j}}\right. \\
& \left.+\frac{2 m_{j}}{n_{i}}+\left(\overline{n_{i}}+\overline{n_{j}}-2\right)\right]^{n_{i} n_{j}} .
\end{aligned}
$$




\section{The Cartesian Product of Graphs:}

The Cartesian product $G_{1} \times G_{2}$ of two graphs $G_{1}$ and $G_{2}$ is the graph with vertex set $V\left(G_{1} \times G_{2}\right)=V\left(G_{1}\right) \times V\left(G_{2}\right)$ and $(u, x)(v, y)$ is an edge of $G_{1} \times G_{2}$ if $u v \in E\left(G_{1}\right)$ and $x=y$, or $u=v$ and $x y \in E\left(G_{2}\right)$. Clearly, $\left|E\left(G_{1} \times G_{2}\right)\right|=\left|E\left(G_{1}\right)\right|\left|V\left(G_{2}\right)\right|+\left|E\left(G_{2}\right)\right|\left|V\left(G_{1}\right)\right|$. In the following theorem, we obtain reformulated NK-index of the Cartesian product of two graphs $G_{1}$ and $G_{2}$. The degree of the vertices of $G_{1} \times G_{2}$ are given by $d_{G_{1} \times G_{2}}(u, v)=$ $d_{G_{1}}(u)+d_{G_{2}}(v)$.

Theorem 3. let $G_{1}$ and $G_{2}$ be two vertex-disjoint connected graphs. Then $\left[N K_{E}\left(G_{1} \times G_{2}\right) \leq\left[\frac{M_{1}\left(G_{1}\right)}{n_{2}}+\frac{4 m_{2}}{n_{2}}-2\right]^{n_{2} m_{1}}\left[\frac{M_{1}\left(G_{2}\right)}{n_{1}}+\frac{4 m_{1}}{n_{1}}-2\right]^{n_{1} m_{2}}\right]$ with equality if and only if both $G_{1}$ and $G_{2}$ are regular graphs.

Proof. We have, from definition of reformulated NK-index

$$
\begin{aligned}
N K_{E}\left(G_{1} \times G_{2}\right)= & \prod_{(a, x)(a, y) \in E\left(G_{1} \times G_{2}\right)}\left[d_{G_{1} \times G_{2}}(a, x)+\right. \\
= & \left.d_{G_{1} \times G_{2}}(b, y)-2\right] \\
& \prod_{(a, x)(a, y), x y \in E\left(G_{2}\right)}\left[d_{G_{1} \times G_{2}}(a, x)\right. \\
& \left.\times d_{G_{1} \times G_{2}}(a, y)-2\right] \\
& +\prod_{(a, x)(b, x), a b \in E\left(G_{1}\right)}\left[d_{G_{1} \times G_{2}}(a, x)\right. \\
& \left.+d_{G_{1} \times G_{2}}(b, x)-2\right] \\
= & C_{1} \times C_{2} .
\end{aligned}
$$

Where $C_{1}$ and $C_{2}$ denote the products of the above terms respectively. Now,

$$
\begin{aligned}
C_{1} & =\prod_{(a, x)(a, y), x y \in E\left(G_{2}\right)}\left[d_{G_{1} \times G_{2}}(a, x)+d_{G_{1} \times G_{2}}(a, y)-2\right] \\
& =\prod_{a \in V\left(G_{1}\right)} \prod_{x y \in E\left(G_{2}\right)}\left[2 d_{G_{1}}(a)+d_{G_{2}}(x)+d_{G_{2}}(y)-2\right] .
\end{aligned}
$$

Now using lemma 1, we have 


$$
\begin{aligned}
C_{1} \leq & {\left[\frac { 1 } { n _ { 1 } m _ { 2 } } \sum _ { a \in V ( G _ { 1 } ) } \sum _ { x y \in E ( G _ { 2 } ) } \left\{2 d_{G_{1}}(a)+\left(d_{G_{2}}(x)+\right.\right.\right.} \\
& \left.\left.\left.d_{G_{2}}(y)\right)-2\right\}\right]^{n_{1} m_{2}} \\
= & {\left[\frac{1}{n_{1} m_{2}}\left\{4 m_{1} m_{2}+n_{1} M_{1}\left(G_{2}\right)-2 n_{1} m_{2}\right\}\right]^{n_{1} m_{2}} } \\
= & {\left[\frac{M_{1}\left(G_{2}\right)}{m_{2}}+\frac{4 m_{1}}{n_{1}}-2\right]^{n_{1} m_{2}} . }
\end{aligned}
$$

Similarly, we have

$$
C_{2} \leq\left[\frac{M_{1}\left(G_{1}\right)}{m_{1}}+\frac{4 m_{2}}{n_{2}}-2\right]^{n_{2} m_{1}} .
$$

From the equality condition of arithmatic-geometric inequality, it is clear that, in the above results equality holds, if and only if both $G_{1}$ and $G_{2}$ are regular graphs. Thus, combining the contribution of $C_{1}$ and $C_{2}$, we get the desired result.

Note that, if $G_{1}$ and $G_{2}$ are $\gamma_{1}$ and $\gamma_{2}$-regular graphs, then $G_{1} \times G_{2}$ be a $\left(r_{1}+r_{2}\right)$-regular graph. Hence, the following corollary follows:

Corollary 2. If $G_{1}$ be a $r_{1}$-regular graph and $G_{2}$ be a $r_{2}$-regular graph, then

$$
N K_{E}\left(G_{1} \times G_{2}\right)=\left\{2\left(r_{1}+r_{2}-1\right)\right\}^{\frac{n_{1} n_{2}}{2}\left(r_{1}+r_{2}\right)} \text {. }
$$

Using above results, the following example follows immediately.

\section{Example 3.}
(i) $N K_{E}\left(C_{n} \times C_{m}\right)=36^{n m}$
(ii) $N K_{E}\left(K_{n} \times K_{m}\right)=\{2(n+m-3)\}^{\frac{n m}{2}(n+m-3)}$.

\section{Corona Product:}

The corona product of two graphs $G_{1}$ and $G_{2}$, denoted by $G_{1} \circ G_{2}$, is the graph obtained by taking one copy of $G_{1}$ and $\left|V\left(G_{1}\right)\right|$ copies of $G_{2}$; and by joining each vertex of i-th copy of $G_{2}$ to the i-th vertex of $G_{1}$, for $1 \leq i \leq\left|V\left(G_{1}\right)\right|$. Thus $\left|V\left(G_{1} \circ G_{2}\right)\right|=\left|V\left(G_{1}\right)\right|+\left|V\left(G_{1}\right)\right|\left|V\left(G_{2}\right)\right|$ and 
$\left|E\left(G_{1} \circ G_{2}\right)\right|=\left|E\left(G_{1}\right)\right|+\left|V\left(G_{1}\right)\right|\left|E\left(G_{2}\right)\right|+\left|V\left(G_{1}\right)\right|\left|V\left(G_{2}\right)\right|$. The degree of the vertices of $G_{1} \circ G_{2}$ is given by

$$
d_{G_{1} \circ G_{2}}(v)=\left\{\begin{array}{l}
d_{G_{1}}(v)+n_{2}, \text { if } v \in V\left(G_{1}\right) \\
d_{G_{2}}(v)+1, \text { if } v \in V\left(G_{2}\right) .
\end{array}\right.
$$

Now, in the following theorem, we derive reformulated NK-index of $G_{1} \circ G_{2}$.

Theorem 4. Let $G_{1}$ and $G_{2}$ be two vertex disjoint connected graphs.

Then

$$
\begin{aligned}
N K_{E}\left(G_{1} \circ G_{2}\right) \leq & {\left[\frac{M_{1}\left(G_{1}\right)}{m_{1}}+2\left(n_{2}-1\right)\right]^{m_{1}} } \\
& {\left[\frac{M_{1}\left(G_{2}\right)}{m_{2}}\right]^{m_{2} n_{1}} \times\left[\frac{2 m_{1}}{n_{1}}+\frac{2 m_{2}}{n_{2}}+\right.} \\
& \left.n_{2}-1\right]^{n_{1} n_{2}}
\end{aligned}
$$

with equality if and only if both $G_{1}$ and $G_{2}$ are regular graphs.

Proof. From definition of reformulated NK-index of a graph, we have

$$
\begin{aligned}
& N K_{E}\left(G_{1} \circ G_{2}\right)=\prod_{u_{i} u_{k} \in E\left(G_{1}\right)}\left(d_{G_{1}}\left(u_{i}\right)+n_{2}+d_{G_{1}}\left(u_{k}\right)\right. \\
& \left.+n_{2}-2\right) \\
& \times \prod_{u_{i} \in V\left(G_{1}\right)} \prod_{v_{j} \in V\left(G_{2}\right)}\left(d_{G_{1}}\left(u_{i}\right)+n_{2}+\right. \\
& \left.d_{G_{2}}\left(v_{j}\right)+1-2\right) \\
& \times \prod_{u_{i} \in V\left(G_{1}\right)} \prod_{v_{i} v_{l} \in E\left(G_{2}\right)}\left(d_{G_{2}}\left(v_{j}\right)+1+\right. \\
& \left.d_{G_{2}}\left(v_{l}\right)+1-2\right) \\
& =P_{1} \times P_{2} \times P_{3}
\end{aligned}
$$

Where $P_{1}, P_{2}$ and $P_{3}$ denote the above three products respectively. Now applying lemma 1 , we can write

$$
P_{1}=\prod_{u_{i} u_{k} \in E\left(G_{1}\right)}\left(d_{G_{1}}\left(u_{i}\right)+d_{G_{1}}\left(u_{k}\right)+2 n_{2}-2\right)
$$




$$
\begin{aligned}
& \leq\left[\frac { 1 } { m _ { 1 } } \sum _ { u _ { i } u _ { k } \in E ( G _ { 1 } ) } \left\{\left(d_{G_{1}}\left(u_{i}\right)+d_{G_{1}}\left(u_{k}\right)+2\left(n_{2}-\right.\right.\right.\right. \\
& 1)\}]^{m_{1}} \\
= & {\left[\frac{M_{1}\left(G_{1}\right)}{m_{1}}+2\left(n_{2}-1\right)\right]^{m_{1}} }
\end{aligned}
$$

Similarly, applying lemma 1, we have

$$
\begin{aligned}
P_{2}= & \left.\prod_{u_{i} \in V\left(G_{1}\right)} \prod_{v_{j} \in V\left(G_{2}\right)}\left(d_{G_{1}}\left(u_{i}\right)+d_{G_{2}}\left(v_{j}\right)\right)+\left(n_{2}-1\right)\right) \\
\leq & {\left[\frac { 1 } { n _ { 1 } n _ { 2 } } \sum _ { u _ { i } \in V ( G _ { 1 } ) } \sum _ { v _ { j } \in V ( G _ { 2 } ) } \left\{\left(d_{G_{1}}\left(u_{i}\right)+d_{G_{2}}\left(v_{j}\right)\right)\right.\right.} \\
& \left.\left.+\left(n_{2}-1\right)\right\}\right]^{n_{1} n_{2}} \\
= & {\left[\frac{1}{n_{1} n_{2}}\left\{2 m_{1} n_{2}+2 m_{2} n_{1}+n_{1} n_{2}\left(n_{2}-1\right)\right\}\right]^{n_{1} n_{2}} }
\end{aligned}
$$

Again, applying lemma 1, we similarly have

$$
\begin{aligned}
P_{3}= & \prod_{u_{i} \in V\left(G_{1}\right)} \prod_{v_{j} v_{l} \in E\left(G_{2}\right)}\left(d_{G_{2}}\left(v_{j}\right)+d_{G_{2}}\left(v_{l}\right)\right) \\
= & {\left[\prod_{v_{j} v_{l} \in E\left(G_{2}\right)}\left(d_{G_{2}}\left(v_{j}\right)+d_{G_{2}}\left(v_{l}\right)\right)\right]^{n_{1}} } \\
& \leq\left[\frac{1}{m_{2}} \sum_{v_{j} v_{l} \in E\left(G_{2}\right)}\left(d_{G_{2}}\left(v_{j}\right)+d_{G_{2}}\left(v_{l}\right)\right)\right]^{n_{1} m_{2}} \\
= & {\left[\frac{M_{1}\left(G_{2}\right)}{m_{2}}\right]^{n_{1} m_{2}} . }
\end{aligned}
$$

From the equality condition of A.M.-G.M. inequality, it is evident that, in the above inequality, equality hold if and only if both $G_{1}$ and $G_{2}$ are regular graphs. Hence, combining $P_{1}, P_{2}$ and $P_{3}$, we get the desired result.

Corollary 3. Let $G_{1}$ be a $r_{1}$-regular graph and $G_{2}$ be a $r_{2}$-regular graph then $\left[N K_{E}\left(G_{1} \circ G_{2}\right)=\left[2\left(r_{1}+n_{2}-1\right)\right]^{\frac{n_{1} r_{1}}{2}}\left[r_{1}+r_{2}+n_{2}-1\right]^{n_{1} n_{2}}\left(2 r_{2}\right)^{\frac{1}{2} n_{1} n_{2} r_{2}}\right.$.]

From the above result the following example follows:

\section{Example 4.}
(i) $N K_{E}\left(C_{n} \circ K_{2}\right)=3^{n} 2^{6 n}$
(ii) $N K_{E}\left(K_{2} \circ C_{n}\right)=2^{4 n+1} n(n+2)^{2 n}$ 


\section{Conclusion}

In this paper, we compute the Narumi-Katayama index of some graph operations such as join of graphs, Cartesian product of graphs and corona product of graphs and consider some special case. From our derived results, we compute this index for some special graphs known as path graph, complete graphs and cycle. For further study, we consider some other graph operations to compute this Narumi-Katayama index.

\section{References}

[1] I. Gutman and N. Trinajstić, "Graph theory and molecular orbitals. Total $\varphi$-electron energy of alternant hydrocarbons", Chemical physics letters, vol. 17, no. 4, pp. 535-538, Dec. 1972, doi: 10.1016/0009-2614(72)85099-1

[2] H. Narumi and M. Katayama, "Simple topological index: a newly devised index characterizing the topological nature of structural isomers of saturated hydrocarbons", Memoirs of the Faculty of Engineering, Hokkaido University, vol. 16, no. 3, pp. 209-214, 1984. [On line]. Available: https:// bit.ly/366Nzw7

[3] Z. Tomovic and I. Gutman, "Narumi-Katayama index of phenylenes", Journal of the Serbian Chemical Society, vol. 66, no. 4, pp. 243-247, 2001, doi: $10.2298 /$ jsc0104243t

[4] R. Todeschini, D. Ballabio, and V. Consonni, "Novel molecular descriptors based on functions of new vertex degrees". En: I. Gutman and B. Furtula (Eds.), Novel molecular structure descriptors-theory and applications. Kragu jevac: University Kragu jevac, 2010, pp. 73-100.

[5] M. Eliasi, A. Iranmanesh, and I. Gutman, "Multiplicative versions of first Zagreb index", MATCH Communications in mathematical and in computer chemistry, vol. 68, no. 1, pp. 217-230, 2012. [On line]. Available: https:/ / bit.ly/33UtOVO

[6] R. Todeschini and V. Consonni, "New local vertex invariants and molecular descriptors based on functions of the vertex degrees", MATCH Communications in mathematical and in computer chemistry, vol. 64, pp.359-372, 2010. [On line]. Available: https:// bit.ly/33YT4dA

[7] I. Gutman, "Multiplicative Zagreb indices of trees", Bulletin of international mathematical virtual institute, vol. 1, pp. 13-19, 2011. [On line]. Available: https:// bit.ly/3j7hkjZ 
[8] K. Xu and H. Hua, "A unified approach to extremal multiplicative Zagreb indices for trees, unicyclic and bicyclic graphs", MATCH Communications in mathematical and in computer chemistry, vol. 68, pp. 241-256, 2012. [On line]. Available: https:// bit.ly/2HuhWCh

[9] J. Liu and Q. Zhang, "Sharp upper bounds for multiplicative Zagreb Indices", MATCH Communications in mathematical and in computer chemistry, vol. 68, pp. 231-240, 2012. [On line]. Available: https:/ / bit.ly/2EzgJbT

[10] S. Mondal, N. De, and A. Pal, "Multiplicative degree based topological indices of nanostar dendrimers", Biointerface research in applied chemistry, vol. 11, no. 1, pp. 7700-7711, Jul. 2021, doi: 10.33263 / briac1 11.77007711

[11] T. Réti and I. Gutman, "Relations between Ordinary and Multiplicative Zagreb Indices", Bulletin of international mathematical virtual institute, vol. 2, pp. 133-140, 2012. [On line]. Available: https:// bit.ly/3cwy8OK

[12] A. Miličević, S. Nikolić and N. Trinajstić, "On reformulated Zagreb indices", Molecular diversity, vol. 8, no. 4, pp. 393-399, Dec. 2004, doi: 10.1023/ B:MODI.0000047504.14261.2a

[13] B. Zhou and N. Trinajstić, "Some properties of the reformulated Zagreb indices", Journal of mathematical chemistry, vol. 48, no. 3, pp. 714-719, Jun. 2010, doi: 10.1007/s10910-010-9704-4

[14] A. Ilić and B. Zhou, "On reformulated Zagreb indices", Discrete applied mathematics, vol. 160, no. 3, pp. 204-209, Feb. 2012, doi: 10.1016/ j.dam.2011.09.021

[15] G. Su, L. Xiong, L. Xu, and B. Ma, "On the maximum and minimum first reformulated Zagreb index of graphs with connectivity at most k", Filomat, vol. 25, no. 4, pp. 75-83, 2011, doi: 10.2298/FIL1104075S

[16] N. De, "Some bounds of reformulated Zagreb indices", Applied mathematical sciences, vol. 6, no. 101, pp. 5505-5012, 2012. [On line]. Available: https:// bit.ly/3cxWqrK

[17] N. De, "Reformulated Zagreb indices of Dendrimers", Mathematica aeterna, vol. 3, no. 2, pp. 133-138. [On line]. Available: https:/ / bit.ly/ 3kPHAzP

[18] S. Mondal, M. A. Ali, N. De, and A. Pal, "Bounds for neighborhood Zagreb index and its explicit expressions under some graph operations", Proyecciones (Antofagasta, On line), vol. 39, no. 4, pp. 799-819, Jul. 2020, doi: 10.22199/ issn.0717-6279-2020-04-0050 
[19] M. Cancan, I. Ahmad, and S. Ahmad, "Molecular descriptors of certain OTIS interconnection networks", Proyecciones (Antofagasta, On line), vol. 39, no. 4, pp. 769-786, Jul. 2020, doi: $10.22199 /$ issn.0717-6279-2020-04-0048

[20] A. J. M. Khalaf, A. Javed, M. K. Jamil, M. Alaeiyan, and M. Reza Farahani, "Topological properties of four types of porphyrin dendrimers", Proyecciones (Antofagasta, On line), vol. 39, no. 4, pp. 979-993, Jul. 2020, doi: 10.22199/ issn.0717-6279-2020-04-0061 